ANNALES

POLONICI MATHEMATICI

$93.1(2008)$

\title{
Mean stability of a stochastic difference equation
}

\author{
by Viorica Mariela Ungureanu (Targu Jiu) and \\ Sui Sun Cheng (Hsinchu)
}

\begin{abstract}
A simple personal saving model with interest rate based on random fluctuation of national growth rate is considered. We establish connections between the mean stochastic stability of our model and the deterministic stability of related partial difference equations. Then the asymptotic behavior of our stochastic model is studied. Although the model is simple, the techniques for obtaining its properties are not, and we make use of the theory of abstract Banach algebras and weighted spaces. It is hoped that our study will lead to more realistic random models.
\end{abstract}

1. Introduction. To motivate our study, let $r(n)$ be the interest rate of growth earned by a nation in the time period $n \in \mathbb{N}=\{0,1,2, \ldots\}$. In real life, $r(n)$ depends on many deterministic or random factors. Here we are concerned with a simple model related to the random fluctuation of the interest rate. More specifically, suppose the interest rate can fluctuate in discrete manners, e.g., $r(n)$ may take values such as $-1 \%,-0.5 \%, 0 \%, 0.5 \%, 1 \%$, $1.5 \%, \ldots$, etc. Then by properly scaling the unit of interest rates, we may assume that $r(n) \in \mathbb{Z}=\{\ldots,-2,-1,0,1,2, \ldots\}$. Let $P_{i}^{n}$ be the probability that the interest rate in the time period $n$ is equal to $i$, that is, $r(n)=i$ (in particular, initially the probability of finding $r(0)=i$ is $P_{i}^{0}$ ). We assume further that at the end of each time interval, the interest rate may remain the same, or may be changed one unit in any direction. Assume that the probability that the interest rate does not change in a given unit of time is $b$, the probability that it decreases is $c$, and the probability that it increases is $a$. In other words, we are assuming that the stochastic process $r(n)$ is a random walk. By Bayes' formula, it is easy to see that the following partial difference equation holds:

$$
P_{i}^{n+1}=a P_{i-1}^{n}+b P_{i}^{n}+c P_{i+1}^{n}, \quad n \in \mathbb{N}, i \in \mathbb{Z} .
$$

2000 Mathematics Subject Classification: 37H10, 93E15.

Key words and phrases: bank saving equation, random walk, partial difference equation, stability. 
Some useful information can be obtained from this equation. First of all, given an initial distribution $\left\{P_{i}^{0}\right\}_{i \in \mathbb{Z}}$, we may calculate

$$
P_{0}^{1} ; P_{-1}^{1}, P_{1}^{1}, P_{0}^{2} ; P_{-2}^{1}, P_{-1}^{2}, P_{1}^{2}, P_{2}^{1}, P_{0}^{3} ; \ldots
$$

successively in a unique manner. Such a unique sequence together with the sequence $\left\{P_{i}^{0}\right\}$ constitutes a solution and is denoted by $\left\{P_{i}^{n}\right\}_{i \in \mathbb{Z}, n \in \mathbb{N}}$. In particular, if $P_{i}^{0}=1$ for $i=i_{0}$ and $P_{i}^{0}=0$ for $i \neq i_{0}$, then the corresponding solution is called a fundamental solution driven by the point $i_{0}$, and all other solutions (the general solution) can be generated by linear combinations of fundamental solutions (see [1], [2]). Note that the fundamental solution $\left\{P_{i}^{n}\right\}$ driven by $i_{0}$ satisfies $P_{i}^{n}=0$ for all $i \in \mathbb{Z} \backslash\left[i_{0}-n, i_{0}+n\right]$ and $n \in \mathbb{N}$.

An important question is the effect of fluctuation of $r(n)$ on personal savings. More precisely, suppose a sum $x(0)=x$ is deposited into a bank account. Then the saving equation is

$$
x(n+1)-x(n)=\widetilde{r}(n) x(n), \quad n \in \mathbb{N},
$$

where $\widetilde{r}(n)$ is the interest rate offered by the bank. In realistic situations, $\widetilde{r}(n)$ depends on $r(n)$ (through decisions of bank managers). We are then led to equations of the form

$$
x(n+1)=A(r(n)) x(n), \quad n \in \mathbb{N},
$$

subject to the condition

$$
x(0)=x \in \mathbb{R},
$$

where $A: \mathbb{Z} \rightarrow \mathbb{R}$ is a bounded function on $\mathbb{Z}$.

There are many questions we can ask about the properties of this stochastic difference equation. For instance, is there a chance for $\{x(n)\}$ to grow without bound, or to die out? In the following, we will study the mean stability of the process $x(n)$, and the results obtained provide answers to some of these questions.

It may seem that our model is too simple to be useful in everyday life. As will be seen, however, the properties of this simple model are by no means simple. We need to first transform our stochastic problems into deterministic ones which involve partial difference equations. To study the latter, we need to employ techniques of abstract Banach algebras and weighted spaces.

After going through such endeavors, we may see that more realistic random models can be built and handled in the near future.

2. Notations and preliminaries. Let $(\Omega, \mathcal{F}, P)$ be a probability space. If $\xi$ is a random variable then we will denote by $E(\xi)$ the expectation (mean) of $\xi$. For any $\sigma$-algebra $\mathcal{G}$ of subsets of $\mathcal{F}, \mathcal{G} \subset \mathcal{F}$ we will denote by $E[\xi \mid \mathcal{G}]$ the conditional expectation (mean) of $\xi$ with respect to $\mathcal{G}$. If $\mathcal{G}$ is 
the $\sigma$-algebra generated by the random variable $\eta$, then we will also use the notation $E[\xi \mid \eta]$ for the conditional expectation of $\xi$ with respect to $\mathcal{G}$.

If $P\{\omega \mid \eta(\omega)=x\}>0$ we will denote by $E[\xi \mid \eta=x]$ the conditional expectation on the event $\eta=x$, and

$$
E[\xi \mid \eta=x]=\frac{1}{P\{\eta(\omega)=x\}} \int \chi_{\{\eta(\omega)=x\}} \xi(\omega) P(d \omega),
$$

while if $P\{\omega \mid \eta(\omega)=x\}=0$ then $E[\xi \mid \eta=x]=0$.

We also denote by $L^{p}(\Omega, \mathcal{F}, P), p \in \mathbb{N}^{*}=\{1,2,3, \ldots\}$, the space of all equivalence classes of real-valued random variables $\xi$ such that $E|\xi|^{p}<\infty$.

We recall that a random walk on $\mathbb{R}$ is a stochastic process $r(n)=\xi_{1}+$ $\cdots+\xi_{n}, n \in \mathbb{N}^{*}, r(0)=0($ or $r(0)=x \in \mathbb{R})$, where $\xi_{1}, \xi_{2}, \ldots$ are real-valued, independent random variables with the same distribution law. In the case when $r(0)=x$ we will say that the random walk $r(n)$ is driven by the point $x$. We denote by $\mathcal{F}_{n}$ the $\sigma$-algebra generated by $\{r(n): 0 \leq k \leq n\}$.

Throughout this paper some or all of the following hypotheses will be assumed.

(P1) $\{r(n)\}_{n \in \mathbb{N}^{*}}$ is a homogeneous Markov chain with state space $\mathbb{Z}$, time space $\mathbb{N}$ and infinite transition matrix $Q=\left(q_{i j}\right)$ where $q_{i j}=$ $P\{r(n+1)=j \mid r(n)=i\}$ is assumed to satisfy the condition $q_{i j}=0$ for all $j \in \mathbb{Z}-\left\{i-m_{0}, \ldots, i, \ldots, i+m_{0}\right\}$, where $m_{0} \in \mathbb{N}^{*}$ is a given number.

(P2) $\{r(n)\}_{n \in \mathbb{N}^{*}}$ is a simple random walk driven by the point $i_{0} \in \mathbb{Z}$, where the transition matrix $Q=\left(q_{i j}\right)$ is assumed to be given by $q_{i, i-1}=c, q_{i i}=b, q_{i, i+1}=a$ and $q_{i j}=0$ otherwise.

(P3) The function $A: \mathbb{Z} \rightarrow \mathbb{R}$ in (2) is bounded on $\mathbb{Z}$, that is, there exist $l, L \in \mathbb{R}$ such that $A(i) \in[l, L]$ for all $i \in \mathbb{Z}$.

It is known from [3] and [4] that

$$
E[\eta(\omega) \mid r(n)]=\sum_{j=-\infty}^{\infty} \chi_{r(n)=j} E[\eta(\omega) \mid r(n)=j]
$$

if $\eta$ is a nonnegative or an integrable random variable on $(\Omega, \mathcal{F}, P)$. Thus

$$
\begin{aligned}
E[\eta(\omega)] & =\sum_{j=-\infty}^{\infty} P(r(n)=j) E[\eta(\omega) \mid r(n)=j] \\
& =\sum_{j=-\infty}^{\infty} P_{j}^{n} E[\eta(\omega) \mid r(n)=j] .
\end{aligned}
$$


In particular, if (P2) holds, then

$$
E[\eta(\omega)]=\sum_{j=n-i_{0}}^{n+i_{0}} P_{j}^{n} E[\eta(\omega) \mid r(n)=j] .
$$

Our problem is to investigate the asymptotic behavior of the mean square of the solutions of the system (2)-(3) under some of the above hypotheses.

\section{Representation theorems for the solutions of discrete time} systems with Markov perturbations. In the following we will assume that (P1) holds.

To the system (2) we associate the random evolution operator $X(n, k)$ defined by $X(k, k)=1$ and

$$
X(n, k)=A(r(n-1)) A(r(n-2)) \cdots A(r(k)), \quad 0 \leq k<n .
$$

In terms of $X(n, k)$, the unique solution of (2) for $n \geq k$ with the initial condition $x(k)=x \in \mathbb{R}$ is $x(n)=X(n, k) x$. It is clear that $X(n, k) \in$ $L^{2}(\Omega, \mathcal{F}, P)$.

We associate to the system (2) the following partial difference equation:

$$
\begin{aligned}
y_{n+1}^{i} & =[A(i)]^{2} \sum_{j=i-m_{0}}^{i+m_{0}} q_{i j} y_{n}^{j}, \quad i \in \mathbb{Z}, n \in \mathbb{N}, \\
y_{0} & =\phi,
\end{aligned}
$$

where $\phi=\left\{\phi^{i}\right\}_{i \in \mathbb{Z}}, \phi^{i}=1$ for all $i \in \mathbb{Z}$. It is clear that the above system has

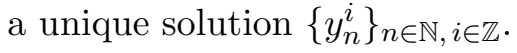

As in [5] where the case of Markov perturbations with finite state space is considered, we can obtain a representation of the mean square of the stochastic process $X(n, k)$ using the solution of the above partial difference equation.

Similar results are obtained in [6] for linear discrete-time stochastic systems with independent random perturbations in Hilbert spaces, and in [7] for linear stochastic differential equations. In [6], it is proved that the covariance operator associated to the solution of the stochastic system satisfies a deterministic linear discrete-time system on a space of nuclear (trace class) operators, and consequently, the stability problem in the stochastic case reduces to the stability problem of the deterministic system.

TheOREM 1. Assume that (P1) holds. If $X(n, k)$ is the random evolution operator associated with the stochastic system (2), and $y=\left\{y_{n}^{i}\right\}_{i \in \mathbb{Z}, n \in \mathbb{N}}$ is the solution of (8)-(9), then for all $i \in \mathbb{Z}$ and $n, k \in \mathbb{N}$ such that $n \geq k$, we have

$$
E\left[X(n, k)^{2} \mid r(k)=i\right]=y_{n-k}^{i}
$$


and

$$
E[X(n, k)]^{2}=\sum_{i=-\infty}^{\infty} P_{i}^{k} y_{n-k}^{i}
$$

where $P_{i}^{k}=P\{r(k)=i\}$.

Proof. Denote by $S$ the set of all functions $g: \mathbb{Z} \rightarrow \mathbb{R}_{+}$and let $f \in S$. Write

$$
(*)=E\left[X(n+1, k)^{2} f(r(n+1)) \mid r(k)=i\right] .
$$

By Lebesgue's monotone convergence theorem,

$$
\begin{aligned}
(*) & =E\left[A(r(n))^{2} X(n, k)^{2} f(r(n+1)) \mid r(k)=i\right] \\
& \left.=\sum_{j=-\infty}^{\infty} E\left[A(r(n))^{2} X(n, k)^{2} \chi_{r(n+1)=j} f(j)\right) \mid r(k)=i\right] .
\end{aligned}
$$

We introduce the function $Y: S \rightarrow S$ defined by

$$
Y(g)(i)=[A(i)]^{2} \sum_{j=i-m_{0}}^{i+m_{0}} q_{i j} g(j), \quad i \in \mathbb{Z} .
$$

It is clear that $Y$ is well defined. Applying the properties of a Markov chain and (5), we get

$$
\begin{aligned}
(*) & \left.=\sum_{j=-\infty}^{\infty} E\left[A(r(n))^{2} X(n, k)^{2} f(j) E\left[\chi_{r(n+1)=j} \mid \mathcal{F}_{n}\right]\right) \mid r(k)=i\right] \\
& \left.=\sum_{j=-\infty}^{\infty} E\left[A(r(n))^{2} X(n, k)^{2} f(j) E\left[\chi_{r(n+1)=j} \mid r(n)\right]\right) \mid r(k)=i\right] \\
& =\sum_{j=-\infty}^{\infty} E\left[A(r(n))^{2} X(n, k)^{2} f(j) q_{r(n) j} \mid r(k)=i\right] \\
& =E\left[X(n, k)^{2} Y(f)(r(n)) \mid r(k)=i\right],
\end{aligned}
$$

where $E\left[\chi_{r(n+1)=j} \mid \mathcal{F}_{n}\right]$ (respectively $E\left[\chi_{r(n+1)=j} \mid r(n)\right]$ ) is the conditional expectation of the random variable $\chi_{r(n+1)=j}$ with respect to the $\sigma$-algebra $\mathcal{F}_{n}$ (respectively the $\sigma$-algebra generated by $r(n)$ ).

So we obtain the recurrence formula

$$
E\left[X(n+1, k)^{2} f(r(n+1)) \mid r(k)=i\right]=E\left[X(n, k)^{2} Y(f)(r(n)) \mid r(k)=i\right] .
$$

Taking $f(i)=1$ for all $i \in \mathbb{Z}$ and iterating we obtain

$$
E\left[X(n+1, k)^{2} \mid r(k)=i\right]=E\left[X(p, k)^{2} Y^{n+1-p}(f)(r(k)) \mid r(k)=i\right]
$$


for all $n+1>p \geq k$. It is easy to see that

$$
Y^{n+1}(f)(i)=Y\left(Y^{n}(f)\right)(i)=[A(i)]^{2} \sum_{j=i-m_{0}}^{i+m_{0}} q_{i j} Y^{n}(f)(j) .
$$

By denoting $Y^{n}(f)(i)=y_{n}^{i}$ for $n \in \mathbb{N}, i \in \mathbb{Z}$, we see that $y_{0}^{i}=Y^{0}(f)(i)=$ $f(i)=1$ for $i \in \mathbb{Z}$ and $y_{n}^{i}$ is the unique solution of (8)-(9). Taking $p=k$ in (12), we obtain (10). Using (6) we obtain (11) and the proof is complete.

Similarly we can prove the following result.

Proposition 1. Assume that (P1) holds. Then for all $i \in \mathbb{Z}$ and $n, k \in \mathbb{N}$ such that $n \geq k$,

$$
E[|X(n, k)| \mid r(k)=i]=\widetilde{z}_{n-k}^{i},
$$

and

$$
E[|X(n, k)|]=\sum_{i=-\infty}^{\infty} P_{i}^{k} \widetilde{z}_{n-k}^{i}
$$

where $\left\{\widetilde{z}_{n}^{i}\right\}_{i \in \mathbb{Z}, n \in \mathbb{N}}$ is the solution of the partial difference equation

$$
\begin{aligned}
\widetilde{z}_{n+1}^{i} & =|A(i)| \sum_{j=i-m_{0}}^{i+m_{0}} q_{i j} \widetilde{z}_{n}^{j}, \quad i \in \mathbb{Z}, n \in \mathbb{N}, \\
\widetilde{z}_{0} & =\phi .
\end{aligned}
$$

4. Stability. Using the results of the above section we can obtain deterministic characterizations of the $p$-mean exponential stability or instability, where $p=1,2$, of the stochastic system (2)-(3).

\section{DeFinition 1.}

(a) We say that the equation (2) is p-mean exponentially stable, where $p=1,2$, iff for any $k \in \mathbb{N}$ there exist $\beta_{k} \geq 1$ and $\alpha_{k} \in(0,1)$ such that

$$
E|X(n, k)|^{p} \leq \beta_{k} \alpha_{k}^{n-k} \quad \text { for all } n \geq k .
$$

(b) We say that the equation (2) is p-mean exponentially instable, where $p=1,2$, iff for any $k \in \mathbb{N}$ there exist $\beta_{k}>0$ and $\alpha_{k}>1$ such that

$$
E|X(n, k)|^{p} \geq \beta_{k} \alpha_{k}^{n-k} \quad \text { for all } n \geq k .
$$

If the $\beta_{k}$ and $\alpha_{k}$ in condition (a) (respectively (b)) of Definition 1 can be taken constant, independent of $k \in \mathbb{N}$, then it is natural to say that the equation (2) is p-mean uniformly exponentially stable (respectively instable).

We remark that it is necessary to take $\beta_{k} \geq 1$, because for $n=k$ in (16) we obtain $E|X(k, k)|^{p} \leq \beta_{k}$, that is, $1 \leq \beta_{k}$. 
We also remark that stability is defined for the more general $X(n, k)$ instead of just for $X(n, 0)$. We motivate this definition by referring to the saving equation, where it is necessary to decide whether money should be deposited into a bank account this year or some time in the future.

The next corollary is a direct consequence of the above definition and of Theorem 1.

Corollary 1. Assume that (P1) holds.

(a) The system (2) is mean square exponentially stable iff for any $k \in \mathbb{N}$ there exist $\beta_{k} \geq 1$ and $\alpha_{k} \in(0,1)$ such that the double sequence $\left\{y_{n}^{i}\right\}$ defined by (8)-(9) satisfies

$$
\sum_{i=-\infty}^{\infty} P_{i}^{k} y_{n}^{i} \leq \beta_{k} \alpha_{k}^{n}, \quad n \in \mathbb{N} .
$$

(b) The system (2) is mean square exponentially instable iff for any $k \in \mathbb{N}$ there exist $\beta_{k}>0$ and $\alpha_{k}>1$ such that the double sequence $\left\{y_{n}^{i}\right\}$ defined by (8)-(9) satisfies

$$
\sum_{i=-\infty}^{\infty} P_{i}^{k} y_{n}^{i} \geq \beta_{k} \alpha_{k}^{n}, \quad n \in \mathbb{N} .
$$

Theorem 2. Assume that (P2) holds and abc $>0$.

(a) The system (2) is mean square exponentially stable iff for any $k \in \mathbb{N}$ there exist $\beta_{k} \geq 1$ and $\alpha_{k} \in(0,1)$ such that the double sequence $\left\{y_{n}^{i}\right\}$ defined by (8)-(9) satisfies

$$
y_{n}^{i} \leq \beta_{k} \alpha_{k}^{n}, \quad\left|i-i_{0}\right| \leq k, n \in \mathbb{N} .
$$

(b) The system (2) is mean square exponentially instable iff there exist $\beta>0$ and $\alpha>1$ such that the component $y_{n}^{i_{0}}$ of the double sequence $\left\{y_{n}^{i}\right\}$ defined by (8)-(9) satisfies

$$
y_{n}^{i_{0}} \geq \beta \alpha^{n}, \quad n \in \mathbb{N} .
$$

Proof. (a) First recall that if (P2) holds, then $P_{i}^{k}=0$ for all $\left|i-i_{0}\right|>k$ and $P_{i}^{k}$ is the coefficient of $x^{i-i_{0}}$ in the expansion of the rational function $\left(a x+b+c x^{-1}\right)^{k}$ (see [2]). Since $a b c>0$, we have $P_{i}^{k} \neq 0$ for all $\left|i-i_{0}\right| \leq k$. By Theorem 1, we get

$$
E[X(n, k)]^{2}=\sum_{i=i_{0}-k}^{i_{0}+k} P_{i}^{k} y_{n-k}^{i}
$$

and statement (a) is an easy consequence of Definition 1 and Theorem 1 .

(b) If there exist $\beta>0$ and $\alpha>1$ such that (21) holds, then for all $k \in \mathbb{N}$ we have 


$$
E[X(n, k)]^{2}=\sum_{i=i_{0}-k}^{i_{0}+k} P_{i}^{k} y_{n-k}^{i} \geq \beta P_{i_{0}}^{k} \alpha^{n-k}
$$

and the conclusion follows by Definition 1. Conversely, if (2) is mean square exponentially instable, then for $k=0$, there exist $\beta>0$ and $\alpha>1$ such that $E[X(n, 0)]^{2} \geq \beta \alpha^{n}$ for all $n \in \mathbb{N}$. Thus $y_{n}^{i_{0}} \geq \beta \alpha^{n}$ for all $n \in \mathbb{N}$ and the conclusion follows.

REMARK 1. (a) By minor modifications of the above proof, the statements of the above theorem remain true even in the cases where $a b c=0$. For example, if $a=0$ and $b c>0$, then $P_{i}^{k}$ is the coefficient of $x^{i-i_{0}}$ in the expansion of $\left(b+c x^{-1}\right)^{k}$. It is clear that $P_{i}^{k} \neq 0$ iff $i_{0}-k \leq i \leq i_{0}$. It is not difficult to see that if we replace the condition $\left|i-i_{0}\right| \leq k$ in (20) with $i \in\left[i_{0}-k, i_{0}\right]$ then statements (a) and (b) of the above theorem stay true in the case where (P2) holds, $a=0$ and $b c>0$.

Analogously if (P2) holds, $c=0$ and $a c>0$, then (a) and (b) hold if we replace $\left|i-i_{0}\right| \leq k$ in (20) with $i \in\left[i_{0}, i_{0}+k\right]$.

(b) Replacing the double sequence $\left\{y_{n}^{i}\right\}$ in the statements of Corollary 1 and Theorem 2 with the double sequence $\left\{\widetilde{z}_{n}^{i}\right\}$ defined by (14) and (15) we obtain necessary and sufficient conditions for the 1-mean exponential stability, respectively instability of (2).

(c) From statement (b) of Theorem 2 and the above remark we deduce that system (2) is $p$-mean exponentially instable, $p=1,2$, iff there exist $\beta>0$ and $\alpha>1$ such that, for all $n \in \mathbb{N}$,

$$
E|X(n, 0)|^{p} \geq \beta \alpha^{n} \text {. }
$$

Corollary 1 and Theorem 2 show that, under (P1) or (P2), stability conditions expressed in terms of the 1-mean or 2-mean of the stochastic process $X(n, k)$ are equivalent to conditions for deterministic partial difference equations. Consequently, we need to study the properties of the solutions of partial difference equations such as (8) or (14).

5. Stability and instability results for linear discrete time systems in an ordered Banach algebra. Let $l_{\infty}(\mathbb{Z})$ be the linear space of all doubly infinite real sequences $y=\left(\ldots, y^{-1}, y^{0}, y^{1}, y^{2}, \ldots\right), y^{i} \in \mathbb{R}, i \in \mathbb{Z}$, that satisfy

$$
\|y\|_{\infty}=\sup _{i \in \mathbb{Z}}\left|y^{i}\right|<\infty .
$$

It is known that $l_{\infty}(\mathbb{Z})$, when endowed with the usual operations, is a Banach space with the norm $\|\cdot\|_{\infty}$. We will denote as before by $\phi=\left\{\phi^{i}\right\}_{i \in \mathbb{Z}}$ the element of $l_{\infty}(\mathbb{Z})$ where each $\phi^{i}$ is 1 . The Banach space $l_{\infty}(\mathbb{Z})$ is a Banach algebra with multiplication $x \cdot y=\left\{x^{i} y^{i}\right\}_{i \in \mathbb{Z}}\left(|x \cdot y|_{\infty} \leq|x|_{\infty}|y|_{\infty}\right)$ and with unit $\phi$. We will denote by $x^{[n]}$ the product $x \cdot \ldots \cdot x$ (n-times), that 
is, $x^{[n]}=\left\{\left(x^{i}\right)^{n}\right\}_{i \in \mathbb{Z}}$. For comparison, note that we have used $y^{i}$ or $(y)^{i}$ to denote the $i$ th component of $y \in l_{\infty}(\mathbb{R})$.

Now we introduce an order on $l_{\infty}(\mathbb{Z})$. We say that $y \geq z$, where $y, z \in$ $l_{\infty}(\mathbb{Z})$, iff $y^{i} \geq z^{i}$ for all $i \in \mathbb{Z}$. If $y \geq 0$, then $y$ is nonnegative. We denote by $\mathcal{K}$ the cone of all nonnegative vectors in $l_{\infty}(\mathbb{Z})$. If $B$ is a Banach algebra we denote by $L(B)$ the Banach space of all bounded linear operators on $B$.

Consider in $l_{\infty}(\mathbb{Z})$ the system

$$
\begin{aligned}
y_{n+1} & =\Gamma\left(y_{n}\right), \quad n \in \mathbb{N}, \\
y_{0} & =\phi,
\end{aligned}
$$

where $\Gamma \in L\left(l_{\infty}(\mathbb{Z})\right)$ satisfies the condition $\Gamma(\mathcal{K}) \subset \mathcal{K}$. It is clear that (23)-(24) has a unique solution given by $y_{n}=\Gamma^{n}(\phi)=\Gamma(\Gamma(\cdots \Gamma(\phi)))$.

REMARK 2. $\Gamma(y) \geq 0$ for all $y \in \mathcal{K}$ and consequently $\Gamma(y) \geq \Gamma(z)$ for all $y \geq z$, where $y, z \in l_{\infty}(\mathbb{Z})$. Also $\|\Gamma(y)\|_{\infty} \geq\|\Gamma(z)\|_{\infty}$ for all $y \geq z, y, z \in \mathcal{K}$.

The following result is analogous to a known result where $H$ is a Hilbert space, $\mathcal{K}_{H}$ is the cone of all nonnegative operators on $H$, and $\Gamma$ is a linear operator on $L(H)$ satisfying $\Gamma\left(\mathcal{K}_{H}\right) \subset \mathcal{K}_{H}$ (see [8]).

Lemma 1. If $\Gamma \in L\left(l_{\infty}(\mathbb{Z})\right)$ and $\Gamma(\mathcal{K}) \subset \mathcal{K}$, then $\|\Gamma\|=\|\Gamma(\phi)\|_{\infty}$.

Proof. For any $y \in l_{\infty}(\mathbb{Z})$, since $-\sup _{i \in \mathbb{Z}}\left|y^{i}\right| \leq y^{i} \leq \sup _{i \in \mathbb{Z}}\left|y^{i}\right|$, we see that $-\|y\|_{\infty} \phi \leq y \leq\|y\|_{\infty} \phi$. Using Remark 2 we get $\Gamma\left(-\|y\|_{\infty} \phi\right) \leq \Gamma(y) \leq$ $\Gamma\left(\|y\|_{\infty} \phi\right)$ and $-\|y\|_{\infty} \Gamma(\phi) \leq \Gamma(y) \leq\|y\|_{\infty} \Gamma(\phi)$. Hence $-\|y\|_{\infty} \Gamma^{i}(\phi) \leq$ $\Gamma^{i}(y) \leq\|y\|_{\infty} \Gamma^{i}(\phi)$ and $\left|\Gamma^{i}(y)\right| \leq\|y\|_{\infty} \Gamma^{i}(\phi)\left(\Gamma^{i}(\phi) \geq 0\right.$ for all $\left.i \in \mathbb{Z}\right)$. Thus $\sup _{i \in \mathbb{Z}}\left|\Gamma^{i}(y)\right| \leq\|y\|_{\infty} \sup _{i \in \mathbb{Z}} \Gamma^{i}(\phi)$ and $\|\Gamma(y)\|_{\infty} \leq\|y\|_{\infty}\|\Gamma(\phi)\|_{\infty}$.

Now it is clear that $\sup _{\|y\|_{\infty}=1}\|\Gamma(y)\|_{\infty} \leq\|\Gamma(\phi)\|_{\infty}$ and $\|\Gamma\| \leq\|\Gamma(\phi)\|_{\infty}$. The other inequality is trivial.

DeFinition 2.

(a) We say that the solution $\left\{y_{n}\right\}$ of $(23)-(24)$ has the property (E) if there exist $\beta>1$ and $\alpha \in(0,1)$ such that

$$
\left\|y_{n}\right\|_{\infty} \leq \beta \alpha^{n} \quad \text { for all } n \in \mathbb{N}
$$

and has the property (IE) if there exist $\beta>0$ and $\alpha>1$ such that

$$
\left\|y_{n}\right\|_{\infty} \geq \beta \alpha^{n} \quad \text { for all } n \in \mathbb{N} .
$$

(b) We say that the $i$ th component $y_{n}^{i}, i \in \mathbb{Z}$, of the solution $\left\{y_{n}\right\}$ of (23)-(24) has the property (CE) if there exist $\beta_{i}>1$ and $\alpha_{i} \in(0,1)$ such that

$$
\left|y_{n}^{i}\right| \leq \beta_{i} \alpha_{i}^{n} \quad \text { for all } n \in \mathbb{N},
$$

and has the property (CIE) if there exist $\beta_{i}>0$ and $\alpha_{i}>1$ such that

$$
\left|y_{n}^{i}\right| \geq \beta_{i} \alpha_{i}^{n} \quad \text { for all } n \in \mathbb{N} .
$$


REMARK 3 .

(a) The solution $\left\{y_{n}\right\}$ of $(23)-(24)$ has the property (E) if there exist $\beta>1$ and $\alpha \in(0,1)$ such that $\left\|\Gamma^{n}(\phi)\right\|_{\infty} \leq \beta \alpha^{n}$ for all $n \in \mathbb{N}$, and has the property (IE) iff there exist $\beta>0$ and $\alpha>1$ such that $\left\|\Gamma^{n}(\phi)\right\|_{\infty} \geq \beta \alpha^{n}$ for all $n \in \mathbb{N}$.

(b) If the solution $\left\{y_{n}\right\}$ of $(23)-(24)$ has the property (E), then every component $y_{n}^{i}, i \in \mathbb{Z}$, of this solution has the property (CE). The converse is not true. On the other hand, if there exist $\beta>1$ and $\alpha \in(0,1)$ such that

$$
\left|y_{n}^{i}\right| \leq \beta \alpha^{n}
$$

for all $i \in \mathbb{Z}, n \in \mathbb{N}$, we deduce that $\left\{y_{n}\right\}$ has the property (E).

(c) If there exists a component $y_{n}^{\hat{i}}, \widehat{i} \in \mathbb{Z}$, of the solution $\left\{y_{n}\right\}$ of (23)-(24) which has the property (CIE), then $\left\{y_{n}\right\}$ has the property (IE). The converse is not true.

The next theorem establishes necessary and sufficient conditions for the exponential stability of the system (23)-(24). In some sense this result is a version of Theorem 17 in [6] which gives a characterization of the uniform exponential stability of a discrete time system of type (23) by using Lyapunov equations of type (26).

THEOREM 3. The following statements are equivalent:

(1) The solution $\left\{y_{n}\right\}$ of (23)-(24) has the property (E).

(2) There exists a unique solution $z \in \mathcal{K}$ of the equation

$$
z=\Gamma(z)+\phi
$$

(3) $r(\Gamma)<1$, where $r(\Gamma)$ denote the spectral radius of $\Gamma$.

Proof. $(1) \Rightarrow(2)$. Assume that (23)-(24) has the property (E). Then $\sum_{k=0}^{\infty} \Gamma^{k}(\phi)$ is convergent in $l_{\infty}(\mathbb{Z})$ (we note that $\Gamma^{0}(\phi)=\phi$ ). Indeed, using Remark 3(a) we deduce

$$
\sum_{k=0}^{\infty}\left\|\Gamma^{k}(\phi)\right\|_{\infty} \leq \sum_{k=0}^{\infty} \beta \alpha^{k}=\beta \frac{1}{1-\alpha}
$$

and the conclusion follows. Now set $z=\sum_{k=0}^{\infty} \Gamma^{k}(\phi)$. Since $\Gamma$ is continuous we have

$$
\Gamma(z)=\Gamma\left(\sum_{k=0}^{\infty} \Gamma^{k}(\phi)\right)=\sum_{k=0}^{\infty} \Gamma^{k+1}(\phi)=z-\phi,
$$

that is, $z$ is a solution of (26). It is clear that since $\phi \geq 0$, we have $\Gamma^{k}(\phi) \geq 0$ for all $k \geq 1$ and $z \geq \phi$. Hence $\|z\|_{\infty} \geq 1$. Now we will prove the uniqueness. Assume that $z, w \in \mathcal{K}$ are two solutions of (26). Then $z-w=\Gamma(z-w)$ and $z-w=\Gamma^{n}(z-w)$ for any $n \in \mathbb{N}$. Since

$$
\|z-w\|_{\infty} \leq\|z\|_{\infty}+\|w\|_{\infty} \leq 2 \max \left\{\|z\|_{\infty},\|w\|_{\infty}\right\}=2 M
$$


we see that $\|z-w\|_{\infty} \leq 2 M\left\|\Gamma^{n}\right\|$. Using the properties of $\Gamma$ we deduce that $\Gamma^{n}(\mathcal{K}) \subset \mathcal{K}$. Applying Lemma 1 we obtain $\left\|\Gamma^{n}\right\|=\left\|\Gamma^{n}(\phi)\right\|_{\infty}$ and consequently $\|z-w\|_{\infty} \leq 2 M \beta \alpha^{n}$ for any $n \in \mathbb{N}$. Letting $n \rightarrow \infty$ yields $\|z-w\|_{\infty}=0$ and $z=w$.

$(2) \Rightarrow(1)$. Assume that $(26)$ has the solution $z \in \mathcal{K}$. We define $f_{n}=\Gamma^{n}(z)$ and we see that

$$
f_{n}=\Gamma^{n}(\Gamma(z)+\phi)=\Gamma^{n+1}(z)+\Gamma^{n}(\phi)=f_{n+1}+\Gamma^{n}(\phi) .
$$

Since $z \geq 0$ we deduce from (26) that $z \geq \phi$ and $\|z\|_{\infty} \geq 1$. Writing $M=2\|z\|_{\infty}<\infty$ we get

$$
\phi \geq \frac{1}{M} z, \quad \Gamma^{n}(\phi) \geq \frac{1}{M} \Gamma^{n}(z)=\frac{1}{M} f_{n}
$$

and

$$
\left(1-\frac{1}{M}\right) f_{n} \geq f_{n+1}, \quad n \in \mathbb{Z} .
$$

Hence $\Gamma^{n}(z) \leq(1-1 / M)^{n} z$ for all $n \in \mathbb{Z}$ and using the inequality $z \geq \phi$ we obtain $\Gamma^{n}(\phi) \leq(1-1 / M)^{n} z$.

Now it is clear that

$$
\left\|\Gamma^{n}(\phi)\right\|_{\infty} \leq\|z\|_{\infty}\left(1-\frac{1}{M}\right)^{n}=M\left(1-\frac{1}{M}\right)^{n} .
$$

Taking $\beta=M$ and $\alpha=1-1 / M$ we deduce that $\left\|y_{n}\right\|_{\infty} \leq \beta \alpha^{n}$ and the conclusion follows.

$(1) \Rightarrow(3)$. Reasoning as above we see that

$$
\left\|\Gamma^{n}\right\|=\left\|\Gamma^{n}(\phi)\right\|_{\infty}<2 \beta \alpha^{n}, \quad \sqrt[n]{\left\|\Gamma^{n}\right\|}<\sqrt[n]{2 \beta \alpha^{n}}
$$

and

$$
\lim _{n \rightarrow \infty} \sqrt[n]{\left\|\Gamma^{n}\right\|} \leq \lim _{n \rightarrow \infty} \sqrt[n]{2 \beta \alpha^{n}}<1
$$

Thus $r(\Gamma)<1$.

$(3) \Rightarrow(1)$. If $r(\Gamma)<1$ then $\lim _{n \rightarrow \infty} \sqrt[n]{\left\|\Gamma^{n}\right\|}=s<1$. We deduce that there exists $\varepsilon>0$ such that $s+\varepsilon=\alpha<1$. Then there exists $k_{0} \in \mathbb{N}$ such that for all $n \geq k_{0}$ we have $\left\|\Gamma^{n}\right\| \leq \alpha^{n}$. If we take

$$
\beta=\max \left\{1,\|\Gamma\| / \alpha, \ldots,\left\|\Gamma^{k_{0}-1}\right\| / \alpha^{k_{0}-1}\right\},
$$

we see that $\left\|\Gamma^{n}\right\| \leq \beta \alpha^{n}$ for all $n \in \mathbb{N}$. Using Lemma 1 again, we obtain $\left\|\Gamma^{n}(\phi)\right\|_{\infty} \leq \beta \alpha^{n}$ and the conclusion follows.

REMARK 4 . Consider the ordered Banach algebra $l_{\infty}$ of all infinite real sequences $v=\left(v^{0}, v^{1}, v^{2}, \ldots\right)$ such that

$$
\|v\|_{\infty}=\sup _{i \in \mathbb{N}}\left|v^{i}\right|<\infty
$$


and $l_{\infty}$ is endowed with the usual operations and the order defined by $y \geq z$ iff $y^{i} \geq z^{i}$ for all $i \in \mathbb{N}$. If we consider the following system on $l_{\infty}$ :

$$
\begin{aligned}
y_{n+1} & =\Gamma_{+}\left(y_{n}\right), \\
y_{0} & =\phi_{+}, \quad \phi_{+}^{i}=1, i \in \mathbb{N},
\end{aligned}
$$

and if we assume $\Gamma_{+} \in L\left(l_{\infty}\right), \Gamma_{+}\left(\mathcal{K}_{+}\right) \subset \mathcal{K}_{+}\left(\mathcal{K}_{+}\right.$is the cone of all nonnegative vectors belonging to $l_{\infty}$ ) then the statements of the above theorem stay true if we replace $\Gamma$ with $\Gamma_{+}, \phi$ with $\phi_{+}$, and $\mathcal{K}$ with $\mathcal{K}_{+}$.

ExAmple 1. Consider $\Gamma: l_{\infty}(\mathbb{Z}) \rightarrow l_{\infty}(\mathbb{Z})$ defined by

$$
(\Gamma(y))^{i}=c_{i}\left(c y^{i-1}+b y^{i}+a y^{i+1}\right), \quad i \in \mathbb{Z},
$$

where $c_{i} \in(0, L)$ for all $i \in \mathbb{Z}$. We will assume that $b>0$ and $\left\{c_{i}\right\}_{i \in \mathbb{Z}}$ has an accumulation point $\eta>1 / b$. It is clear that $\Gamma \in L\left(l_{\infty}(\mathbb{Z})\right)$. We will investigate whether the solution $y_{n}$ of $(23)-(24)$ has the property $(\mathrm{E})$.

Under the above assumptions, the equation (26) can be written as

$$
c_{i}\left(c y^{i-1}+b y^{i}+a y^{i+1}\right)+1=y^{i} .
$$

Assume that (27) has a unique nonnegative solution on $l_{\infty}(\mathbb{Z})$. Let $\left\{i_{n}\right\}_{n \in \mathbb{N}}$ be an integer sequence such that $c_{i_{n}} \rightarrow \eta$ as $n \rightarrow \infty$. Since $y \in l_{\infty}(\mathbb{Z})$ and $y \geq 0$, we see that $\left\{y^{i_{n}}\right\}_{n \in \mathbb{N}}$ is bounded and there exists a subsequence $\left\{y^{i_{n_{k}}}\right\}$ converging to $\tau \geq 1$ (we recall that $y \geq \phi$ ). Analogously there are subsequences $\left\{y^{i_{p_{k}}-1}\right\},\left\{y^{i_{p_{k}}}\right\},\left\{y^{i_{p_{k}}+1}\right\}$ converging to $\tau_{-1}, \tau_{0}, \tau_{1}$ respectively. Then $\eta c \tau_{-1}+(\eta b-1) \tau_{0}+\eta a \tau_{1}+1=0$, which is impossible.

Hence (26) cannot have a solution in $\mathcal{K}$. By Theorem 3, the solution of (23)-(24) does not have the property (E).

We note that if in the above example $c_{i} \in(0, s)$ where $s<1$, then $\|\Gamma(\phi)\|_{\infty}=\sup _{i \in \mathbb{Z}} c_{i} \leq s<1$. Lemma 1 implies that $\|\Gamma\| \leq s<1$ and consequently $r(\Gamma)<1$. In this situation the solution of $(23)-(24)$ has the property (E) according Theorem 3.

The following result provides a characterization of the property (IE) of the solution of the system (23)-(24).

THEOREM 4. The solution of the system (23)-(24) has the property (IE) iff $r(\Gamma)>1$.

Proof. Assume that there exist $\beta>0$ and $\alpha>1$ such that $\left\|y_{n}\right\|_{\infty} \geq \beta \alpha^{n}$ for all $n \in \mathbb{N}$. Since $y_{n}=\Gamma^{n}(\phi)$, we use Lemma $1\left(\left\|\Gamma^{n}(\phi)\right\|_{\infty}=\left\|\Gamma^{n}\right\|\right)$ to deduce that $\left\|\Gamma^{n}\right\| \geq \beta \alpha^{n}$ for all $n \in \mathbb{N}$. Hence $\sqrt[n]{\left\|\Gamma^{n}\right\|} \geq \sqrt[n]{\beta \alpha^{n}}$ for all $n \in \mathbb{N}$ and passing to the limit we get $r(\Gamma)>1$.

Conversely, if $r(\Gamma)>1$, then $\lim _{n \rightarrow \infty} \sqrt[n]{\left\|\Gamma^{n}\right\|}=t>1$ and there exists $\varepsilon>0$ such that $t-\varepsilon>1$. Hence there exists $N_{\varepsilon} \in \mathbb{N}$ such that $\sqrt[n]{\left\|\Gamma^{n}\right\|} \geq t-\varepsilon$ for all $n \geq N_{\varepsilon}$. We take $\alpha=t-\varepsilon$ and

$$
\beta=\min \left\{1,\|\Gamma\| / \alpha,\left\|\Gamma^{2}\right\| / \alpha^{2}, \ldots,\left\|\Gamma^{N_{e}-1}\right\| / \alpha^{N_{\varepsilon}-1}\right\},
$$


and it follows that $\left\|\Gamma^{n}\right\| \geq \beta \alpha^{n}$ for all $n \in \mathbb{N}$. We note that $\beta \neq 0$, otherwise there exists $k<N_{\varepsilon}, k \in \mathbb{N}$, such that $\Gamma^{k}=0$, which contradicts the assumption $r(\Gamma)>1$. Using Lemma 1 again, we get the conclusion.

Since the property (IE) of the solution of (23)-(24) does not guarantee the property (CIE) (see Remark 3(c)), we will investigate the behavior of the components $y_{n}^{i}, i \in \mathbb{Z}$, of the solution of (23)-(24). The following result gives sufficient conditions for some component $y_{n}^{i}$ of $y_{n}$ to have the property (CIE).

Proposition 2. Let $\left\{y_{n}\right\}_{n \in \mathbb{N}}$, where $y_{n}=\left(\ldots, y_{n}^{-1}, y_{n}^{0}, y_{n}^{1}, \ldots\right)$, be the solution of (23)-(24). Assume that there exist $\alpha>0$ and $\delta>0$ such that $\inf _{i \in \mathbb{Z}}(\Gamma(\phi))^{i}>\alpha$ and $\Gamma(y) \geq \delta \Gamma(\phi) y$. Then

(a) $y_{n}^{i} \geq\left(\gamma(\Gamma(\phi))^{i}\right)^{n}$ for all $n \in \mathbb{N}$ and $i \in \mathbb{Z}$, where $\gamma$ is defined by

$$
\gamma=\inf _{n \in \mathbb{N}^{*}, i \in \mathbb{Z}} \frac{\left(\Gamma\left((\Gamma(\phi))^{[n]}\right)\right)^{i}}{\left((\Gamma(\phi))^{i}\right)^{n+1}}, \quad \gamma \in\left[\delta,(\|\Gamma\| / \alpha)^{2}\right] .
$$

(b) In addition, if there exists $\widehat{i} \in \mathbb{Z}$ such that $\gamma \Gamma^{\hat{i}}(\phi)>1$, then the component $y_{n}^{\hat{i}}$ of $y_{n}$ has the property (CIE).

Proof. (a) Since $(\Gamma(\phi))^{[n]} \in l_{\infty}(\mathbb{Z})$, we see that $\Gamma\left((\Gamma(\phi))^{[n]}\right)$ is well defined. Using the hypothesis we deduce that there exists $\alpha>0$ such that $(\Gamma(\phi))^{i}>\alpha$ for all $i \in \mathbb{Z}$ and

$$
\Gamma\left((\Gamma(\phi))^{[n]}\right) \geq \delta \Gamma(\phi)(\Gamma(\phi))^{[n]} .
$$

Hence

$$
\frac{\left(\Gamma\left((\Gamma(\phi))^{[n]}\right)\right)^{i}}{\left((\Gamma(\phi))^{i}\right)^{n+1}} \geq \delta
$$

On the other hand,

$$
\gamma \leq \frac{(\Gamma(\Gamma(\phi)))^{i}}{\left((\Gamma(\phi))^{i}\right)^{2}} \leq\left(\frac{\|\Gamma\|}{\alpha}\right)^{2}
$$

and so we have proved that $\gamma \in\left[\delta,(\|\Gamma\| / \alpha)^{2}\right]$.

We will prove by induction on $n \in \mathbb{N}$ that $y_{n}^{i} \geq\left(\gamma(\Gamma(\phi))^{i}\right)^{n}$ for the solution of (23)-(24). For $n=0$ this is obviously true. Assume that $y_{n}^{i} \geq$ $\left(\gamma(\Gamma(\phi))^{i}\right)^{n}$. Then $y_{n} \geq \gamma^{n}(\Gamma(\phi))^{[n]}$ and

$$
\begin{aligned}
y_{n+1}^{i} & =\left(\Gamma\left(y_{n}\right)\right)^{i} \geq \gamma^{n}\left(\Gamma\left((\Gamma(\phi))^{[n]}\right)\right)^{i}=\gamma^{n} \frac{\left(\Gamma\left((\Gamma(\phi))^{[n]}\right)\right)^{i}}{\left((\Gamma(\phi))^{i}\right)^{n+1}}\left((\Gamma(\phi))^{i}\right)^{n+1} \\
& \geq \gamma^{n+1}\left((\Gamma(\phi))^{i}\right)^{n+1}=\left(\gamma(\Gamma(\phi))^{i}\right)^{n+1} .
\end{aligned}
$$

The conclusion follows.

(b) We take $\alpha_{\hat{i}}=\gamma \widehat{\Gamma^{\hat{i}}}(\phi)$ and $\beta_{\hat{i}}=1$ and we get the conclusion.

Next we will obtain conditions which ensure that every component of the solution of (23)-(24) has the property (CE) although the solution of 
(23)-(24) does not necessarily possess the property (E). Let us consider the linear space $l_{h}$ of all infinite sequences $y=\left\{y^{i}\right\}_{i \in \mathbb{Z}}$ such that

$$
\|y\|_{h}=\sum_{i=-\infty}^{\infty} \frac{\left|y^{i}\right|}{h^{|i|}}<\infty,
$$

where $h \in \mathbb{R}$ and $h>1$. It is easy to see that $l_{h}$ is a Banach space with the norm $\|\cdot\|_{h}$ introduced above.

Since $l_{\infty}(\mathbb{Z}) \subset l_{h}$, it is natural to ask if a solution of $(23)-(24)$ in $l_{\infty}(\mathbb{Z})$ has the property $(\mathrm{E})$ in $\|\cdot\|_{h}$, that is, if there exist $\beta>1$ and $\alpha \in(0,1)$ such that $\left\|y_{n}\right\|_{h} \leq \beta \alpha^{n}$ for all $n \in \mathbb{N}$.

We note that if this is so, then for all $i \in \mathbb{Z}$ there exist $\beta_{i}=\beta h^{|i|}$ and $\alpha_{i}=\alpha$ such that $\left|y_{n}^{i}\right| \leq \beta_{i} \alpha_{i}^{n}$ for all $n \in \mathbb{N}$.

Assume that $\Gamma \in L\left(l_{h}\right)$ and $\eta \in l_{h}$. Then there exists a unique solution $y_{n}(\eta) \in l_{h}$ of $(23)$ with the initial condition $y_{0}=\eta$.

Recall that the equation (23) is said to be stable if $\lim _{\eta \rightarrow 0} \sup _{n \in \mathbb{N}} y_{n}(\eta)$ $=0$; asymptotically stable if it is stable and $\lim _{n \rightarrow \infty} y_{n}(\eta)=0$ for all $\eta \in l_{h}$; and exponentially stable if there exist $\beta>1$ and $\alpha \in(0,1)$ such that $\left\|y_{n}(\eta)\right\|_{h} \leq \beta \alpha^{n}\|\eta\|_{h}$ for all $\eta \in l_{h}$ and $n \in \mathbb{N}$.

The following lemma is a result of Lyapunov type.

Lemma 2. Let $\eta \in l_{h}$ and $y_{n}(\eta)$ be the unique solution of (23) with the initial condition $y_{0}=\eta$. Assume that $\left\{V_{n}\right\}_{n \in \mathbb{N}}$ is a sequence of real continuous functions defined on $l_{h}$ satisfying:

(1) there exist $\alpha, \beta>0$ such that $\alpha\|\eta\|_{h} \leq V_{n}(\eta) \leq \beta\|\eta\|_{h}$ for all $n \in \mathbb{N}$ and $\eta \in l_{h}$,

(2) there exists a nonnegative sequence $\left\{g_{n}\right\}_{n \in \mathbb{N}}$ and a positive number $Q$ such that $0 \leq g_{n}<Q$ for all $n \in \mathbb{N}, \sum_{n=0}^{\infty} g_{n}=\infty$ and

$$
V_{n+1}\left(y_{n+1}(\eta)\right) \leq V_{n}\left(y_{n}(\eta)\right)-g_{n}\left\|y_{n}(\eta)\right\|_{h}, \quad \eta \in l_{h}, n \in \mathbb{N} .
$$

Then the system (23) is asymptotically stable. Moreover, if $g_{n}=g$ for all $n \in \mathbb{N}$, then (23) is exponentially stable.

Proof. For simplicity, denote $y_{n}(\eta)$ by $y_{n}$. We have

$$
V_{n+1}\left(y_{n+1}\right) \leq V_{0}(\eta)-\sum_{k=0}^{n} g_{k}\left\|y_{k}\right\|_{h}
$$

and

$$
\sum_{n=0}^{\infty} g_{n}\left\|y_{n}\right\|_{h} \leq V_{0}(\eta)<\infty .
$$

It is clear that we cannot find $M>0$ and $N_{M} \in \mathbb{N}$ such that $\left\|y_{n}\right\|_{h} \geq M$ 
for all $n \geq N_{M}$, because otherwise

$$
\sum_{n=0}^{\infty} g_{n}\left\|y_{n}\right\|_{h} \geq M \sum_{n=N_{M}}^{\infty} g_{n}=\infty
$$

and we obtain a contradiction. Thus for all $k \in \mathbb{N}$, there exists $n_{k} \in \mathbb{N}$ such that $\left\|y_{n_{k}}\right\|_{h} \leq 1 / k$. Hence a subsequence converges to 0 . On the other hand, $0 \leq V_{n+1}\left(y_{n+1}\right) \leq V_{n}\left(y_{n}\right)$ and so $V_{n}\left(y_{n}\right)$ is a decreasing sequence, which converges to $l \in \mathbb{R}_{+}$. Since

$$
V_{n_{k}+1}\left(y_{n_{k}+1}\right) \leq\left(\beta-g_{n_{k}}\right)\left\|y_{n_{k}}\right\|_{h}
$$

letting $k \rightarrow \infty$ we obtain $l=0$. Letting $n \rightarrow \infty$ in $V_{n}\left(y_{n}\right) \geq \alpha\left\|y_{n}\right\|_{h}$ we see that $\left\|y_{n}\right\|_{h} \rightarrow 0$ as $n \rightarrow \infty$. On the other hand, $\alpha\left\|y_{n}\right\|_{h} \leq V_{n}\left(y_{n}\right) \leq V_{0}(\eta)$ and

$$
\lim _{\eta \rightarrow 0} \sup _{n \in \mathbb{N}} y_{n}(\eta) \leq \lim _{\eta \rightarrow 0} \frac{V_{0}(\eta)}{\alpha}=0
$$

and the first assertion of the proposition follows.

If $g_{n}=g$ for all $n \in \mathbb{N}$, then $g \leq \beta$ and

$$
V_{n+1}\left(y_{n+1}\right) \leq V_{n}\left(y_{n}\right)-g\left\|y_{n}\right\|_{h} \leq \gamma V_{n}\left(y_{n}\right),
$$

where $\gamma=\max \{1-g / \beta, 1 / 3\}$. Thus $V_{n}\left(y_{n}\right) \leq \gamma^{n} V_{0}(\eta) \leq \beta \gamma^{n}\|\eta\|_{h}$ and using (1) we obtain the conclusion. The proof is complete.

Let us consider the system (8)-(9) in $l_{h}$ and

Example 2. Assume that (P3) holds, $l>0$ and $A(0)=1$, and let $a, b, c$ be real nonnegative numbers satisfying $a+b+c=1, c>a>0$ and $\sqrt{c}-\sqrt{a} \geq 10^{-1}$. In this example, the real numbers $l$ and $L$ are those introduced in (P3). We consider the linear operator $\Gamma: l_{h} \rightarrow l_{h}$ given by

$$
(\Gamma(y))^{i}=[A(i)]^{2}\left(c y^{i-1}+b y^{i}+a y^{i+1}\right)
$$

for $i \in \mathbb{Z}$. It is clear that $\Gamma \in L\left(l_{h}\right)$ and $\Gamma(\mathcal{K}) \subset \mathcal{K}$. We also introduce the mappings $M, m:(1, \infty) \rightarrow \mathbb{R}_{+}$defined by

$$
M(h)=\frac{1}{a h+c / h+b+10^{-2}}, \quad m(h)=\frac{1}{c h+a / h+b+10^{-2}} .
$$

Assume that there exists $h>1$ such that

$$
\bar{M} \leq M(h), \quad \bar{m} \leq m(h) \quad \text { and } \quad \frac{h-1}{h}(a+c)>10^{-2},
$$

where $\bar{m}=\sup _{i \in \mathbb{Z}, i<0}[A(i)]^{2}$ and $\bar{M}=\sup _{i \in \mathbb{Z}, i>0}[A(i)]^{2}$. We will prove that (23) is exponentially stable in $\|\cdot\|_{h}$.

We consider the bounded linear operator $C: l_{h} \rightarrow l_{h}$ defined by $(C y)^{i}=$ $[1 / A(i)]^{2} y^{i}$ for $i \in \mathbb{Z}$. The function $V: l_{h} \rightarrow \mathbb{R}_{+}$defined by

$$
V(y)=\|C y\|_{h}, \quad y \in l_{h},
$$


is continuous and the sequence $\left\{V_{i}=V\right\}_{i \in \mathbb{N}}$ satisfies condition (1) of Lemma 2, where $\alpha=1 / L^{2}$ and $\beta=1 / l^{2}$. Furthermore, setting $\sigma_{+}^{n}=\sum_{i=1}^{\infty}\left|y_{n}^{i}\right| / h^{i}$ and $\sigma_{-}^{n}=\sum_{i=-\infty}^{-1}\left|y_{n}^{i}\right| / h^{|i|}$, we get

$$
\begin{aligned}
\left\|V\left(y_{n+1}\right)\right\|_{h} \leq & \left(a h+\frac{c}{h}+b\right) \sigma_{+}^{n}+\left(\frac{a+c}{h}+b\right)\left|y_{n}^{0}\right|+\left(c h+\frac{a}{h}+b\right) \sigma_{-}^{n} \\
\leq & \bar{M}\left[\left(a h+\frac{c}{h}+b\right)+10^{-2}\right] \frac{\sigma_{+}^{n}}{\bar{M}} \\
& +\bar{m}\left[c h+\frac{a}{h}+b+10^{-2}\right] \frac{\sigma_{-}^{n}}{\bar{m}}+\left|y_{n}^{0}\right|-10^{-2}\left(\sigma_{+}^{n}+\sigma_{-}^{n}+\left|y_{n}^{0}\right|\right) \\
\leq & \sum_{i=1}^{\infty} \frac{1}{[A(i)]^{2}} \frac{\left|y_{n}^{i}\right|}{h^{i}}+\sum_{i=-\infty}^{-1} \frac{1}{[A(i)]^{2}} \frac{\left|y_{n}^{i}\right|}{\left.h\right|^{i} \mid}+\left|y_{n}^{0}\right|-10^{-2}\left\|y_{n}\right\|_{h} \\
= & \left\|V\left(y_{n}\right)\right\|_{h}-10^{-2}\left\|y_{n}\right\|_{h} .
\end{aligned}
$$

Thus condition (2) of Lemma 2 is satisfied with $g_{n}=10^{-2}$ for $n \in \mathbb{N}$. Then $\left\|y_{n}\right\|_{h} \leq(L / l)^{2} \gamma^{n}$, where $\gamma=\max \left\{1-l^{2} / 100,1 / 3\right\}$. The proof is complete.

Now we assume that the stochastic equation (2)-(3) is the saving equation of a sum $x$ deposited into a bank account, (P2) holds and $i_{0}=0$. Then (8)-(9) is the deterministic system associated with the mean square stability problem of (2)-(3). We note that (8) can be written as (23) with $\Gamma \in L\left(l_{h}\right)$ defined by (29). As a numerical example we consider the situation where $c=0.8, b=0.1, a=0.1, A(0)=1, L^{2}=1.04$ and $l^{2}=0.86$. Let us adopt the notation introduced in the above example. The graphs of the functions $M(\cdot), m(\cdot)$ defined above are depicted below.

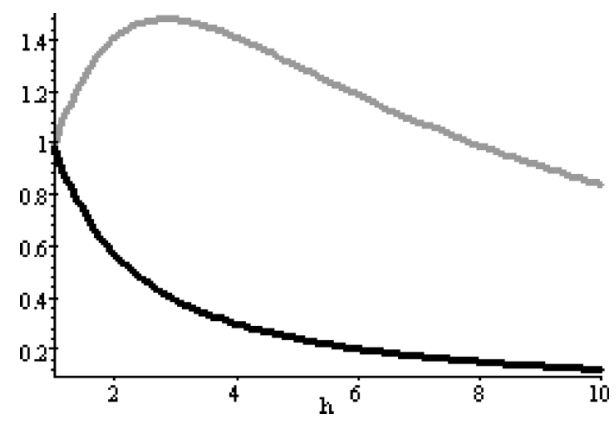

We deduce from the above example that if there exists $h>1$ such that $\bar{M} \leq M(h), \bar{m} \leq m(h)$ and $(h-1)(a+c) / h>10^{-2}$, then the system (8) is exponentially stable. For example if $h=1.2$, then $M(h)=1.1152$, $m(h)=0.86705$ and $(a+c)(h-1) / h=0.15>10^{-2}$. Thus if $\bar{M} \leq 1.115$ and $\bar{m} \leq 0.867$, then $y_{n}^{i} \leq \frac{10.4}{8.6}(0.991)^{n}(1.2)^{i}$ for all $i \in \mathbb{Z}$ and $n \in \mathbb{N}$. Thus the system is exponentially stable. 
Hence, $E[X(n, 0)]^{2}=y_{n}^{0} \leq \frac{10.4}{8.6}(0.991)^{n}$ and we deduce that, under the above hypotheses, there is a good chance to loose 0.025 of the original sum in 25 units of time (for example a unit could be a month).

6. Stability criteria for the stochastic system. Throughout this section we will assume that (P3) holds. Consider the systems (8)-(9) and $(14)-(15)$ in the Banach space $l_{\infty}(\mathbb{Z})$. We see that (8) can be written as

$$
y_{n+1}=\Gamma_{2}\left(y_{n}\right)
$$

where $\Gamma_{2}: l_{\infty}(\mathbb{Z}) \rightarrow l_{\infty}(\mathbb{Z})$ is defined by

$$
\left(\Gamma_{2}(y)\right)^{i}=[A(i)]^{2} \sum_{j=i-m_{0}}^{i+m_{0}} q_{i j} y^{j}, \quad i \in \mathbb{Z} .
$$

It is a simple exercise to see that $\Gamma_{2} \in L\left(l_{\infty}(\mathbb{Z})\right)\left(\right.$ since $\left.\left\|\Gamma_{2}\right\| \leq \max \left\{l^{2}, L^{2}\right\}\right)$. Analogously, (14) can be written as

$$
y_{n+1}=\Gamma_{1}\left(y_{n}\right)
$$

where $\Gamma_{1} \in L\left(l_{\infty}(\mathbb{Z})\right)$ is defined by

$$
\left(\Gamma_{1}(y)\right)^{i}=|A(i)| \sum_{j=i-m_{0}}^{i+m_{0}} q_{i j} y^{j}, \quad i \in \mathbb{Z},\left\|\Gamma_{1}\right\|=\max \{|l|,|L|\} .
$$

It is easy to verify that $\Gamma_{p}(\mathcal{K}) \subset \mathcal{K}, p=1,2$, where $\mathcal{K}$ is the cone of all nonnegative elements of $l_{\infty}(\mathbb{Z})$. If we define $\Gamma_{1}, \Gamma_{2}$ on $l_{h}$ we see that $\Gamma_{1}, \Gamma_{2} \in$ $L\left(l_{h}\right)$.

Theorem 5. Assume that (P1) and (P3) hold. If $r\left(\Gamma_{p}\right)<1$ then the system (2) is p-mean uniformly exponentially stable for $p=1,2$. (Here the spectral radius is computed with respect to the space $L\left(l_{\infty}(\mathbb{Z})\right)$.)

Proof. Assume that $r\left(\Gamma_{2}\right)<1$. Using Theorem 3 and Remark 3(a), we deduce that there exist $\beta>1$ and $\alpha \in(0,1)$ such that $y_{n}^{i} \leq \beta \alpha^{n}$ for all $i \in \mathbb{Z}$. Then $\sum_{i=-\infty}^{\infty} P_{i}^{k} y_{n}^{i} \leq \beta \alpha^{n}$ for all $n \geq k \geq 0$ and the conclusion follows by Corollary 1 (a).

Assume that $r\left(\Gamma_{1}\right)<1$. Reasoning as above we deduce that there exist $\beta>1$ and $\alpha \in(0,1)$ such that $\widetilde{z}_{n}^{i} \leq \beta \alpha^{n}$ for all $i \in \mathbb{Z}$. Using Remark 1(b) we deduce the conclusion.

The following proposition is a direct consequence of Theorems 2, 4 and Remarks 1(b), 3(c) and offers necessary conditions for the exponential instability of the stochastic equation (2).

Proposition 3. Assume that (P2) and (P3) hold. If (2) is p-mean exponentially instable then $r\left(\Gamma_{p}\right)>1, p=1,2$. 
Before we state the next result, note that

$$
\left(\Gamma_{2}(y)\right)^{i}=[A(i)]^{2} \sum_{j=i-m_{0}}^{i+m_{0}} q_{i j} y^{j} \geq[A(i)]^{2} q_{i i} y^{i}=q_{i i}\left(\Gamma_{2}(\phi) y\right)^{i}
$$

and analogously we can prove that

$$
\left(\Gamma_{1}(y)\right)^{i} \geq q_{i i}\left(\Gamma_{1}(\phi) y\right)^{i} .
$$

Let us replace $\Gamma$ with $\Gamma_{p}, p=1,2$, in (28). We obtain the following formulas for $\gamma_{p}$ :

$$
\gamma_{2}=\inf _{n \in \mathbb{N}^{*}, i \in \mathbb{Z}} \frac{\sum_{j=i-m_{0}}^{i+m_{0}} q_{i j}[A(j)]^{2 n}}{[A(i)]^{2 n}}, \quad \gamma_{1}=\inf _{n \in \mathbb{N}^{*}, i \in \mathbb{Z}} \frac{\sum_{j=i-m_{0}}^{i+m_{0}} q_{i j}|A(j)|^{n}}{|A(i)|^{n}} .
$$

Theorem 6. Assume that (P1), (P3) hold, $\inf _{i \in \mathbb{Z}}|A(i)|>0$ and there exists $\delta>0$ such that $q_{i i} \geq \delta$.

(i) If there exists $s>1$ such that $\gamma_{p}[A(i)]^{2} \geq s$ for all $i \in \mathbb{Z}$, then the system (2) is p-uniformly exponentially instable, $p=1,2$.

(ii) In addition, if (P2) holds, abc $>0$ and $\gamma_{p}\left[A\left(i_{0}\right)\right]^{2}>1$, then the system (2) is p-mean uniformly exponentially instable, $p=1,2$.

Proof. We only prove the statements for $p=2$ because the proof for $p=1$ is similar.

(i) Using Proposition 2 we deduce that $y_{n}^{i} \geq s^{n}$ for all $i \in \mathbb{Z}$. Since $\sum_{i=-\infty}^{\infty} P_{i}^{k} y_{n}^{i} \geq s^{n}$, the conclusion follows by Corollary $1(\mathrm{~b})$.

(ii) The conclusion follows by Proposition 2 and Theorem 2(b).

EXAmple 3. Assume that (P2) holds, $i_{0}=0$ and $a b c>0, b+a>1 / 2$. We write $[A(i)]^{2}=c_{i}$ and we also assume that $c_{i}=1 / 2$ for all $i<0$, and $c_{i}=2$ for $i \in \mathbb{N}$. Then $\Gamma_{2}: l_{\infty}(\mathbb{Z}) \rightarrow l_{\infty}(\mathbb{Z})$ and $\left(\Gamma_{2}(y)\right)^{i}=c_{i}\left(c y^{i-1}+b y^{i}+a y^{i+1}\right)$ for $i \in \mathbb{Z}$. Since

$$
\frac{\left(\Gamma\left((\Gamma(\phi))^{[n]}\right)\right)^{i}}{\left((\Gamma(\phi))^{i}\right)^{n+1}}= \begin{cases}1, & |i|>1 \text { and } i=1, \\ c+b+a\left(4^{n}\right), & i=-1, \\ c\left(1 / 4^{n}\right)+b+a, & i=0,\end{cases}
$$

and $\gamma_{2}=b+a$, and since $\gamma_{2}[A(0)]^{2}=2(a+b)>1$, we use statement (ii) of the above theorem to deduce that the system (2) is mean square uniformly exponentially instable. Moreover since $y_{n}^{0} \geq[2(a+b)]^{n}$ for all $n \in \mathbb{N}$, we deduce that $y_{n}$ has the property (IE) and consequently $r\left(\Gamma_{2}\right)>1$.

Proposition 4. Assume that (P3) holds.

(a) If (P1) holds and there exist $M_{0}>0$ and $h>1$ such that $P_{i}^{0} h^{|i|} \leq$ $M_{0}$ for all $i \in \mathbb{Z}$, and $\lim _{n \rightarrow \infty}\left\|y_{n}\right\|_{h}=0$, then for any $k \in \mathbb{N}$,

$$
\lim _{n \rightarrow \infty} E[X(n, k)]^{2}=0 \text {. }
$$


(b) If (P1) is satisfied and there exist $M_{0}>0$ and $h>1$ such that $P_{i}^{0} h^{|i|} \leq M_{0}$ for all $i \in \mathbb{Z}$ and the system (30) is exponentially stable in $l_{h}$, then the system (2) is mean square exponentially stable.

(c) If (P2) holds and there exists $h>1$ such that the system (30) is exponentially stable in $l_{h}$, then the system (2) is mean square exponentially stable.

Proof. (a) Using the hypotheses we see that $P_{i}^{0} \leq\left(1 / h^{i}\right) M_{0}$ and $P_{i}^{0} \leq$ $h^{i} M_{0}$ for all $i \in \mathbb{Z}$. Now, we apply Theorem 4.4 in [2] to deduce that $P_{i}^{k} \leq$ $M_{0}(1 / h)^{i}(a h+b+c / h)^{k}$ and $P_{i}^{k} \leq M_{0} h^{i}(a / h+b+c h)^{k}$ for all $i \in \mathbb{Z}$ and $k \in \mathbb{N}$. Hence $P_{i}^{k} h^{|i|} \leq M_{k}$, where

$$
M_{k}=M_{0} \max \left\{(a h+b+c / h)^{k},(a / h+b+c h)^{k}\right\} .
$$

We further note that for all $n, k \in \mathbb{N}$ with $n>k$,

$$
E[X(n, k)]^{2}=\sum_{i=-\infty}^{\infty} P_{i}^{k} h^{|i|} \frac{y_{n-k}^{i}}{h^{|i|}} \leq M_{k} \sum_{i=-\infty}^{\infty} \frac{y_{n-k}^{i}}{h^{|i|}}=M_{k}\left\|y_{n-k}\right\|_{h} .
$$

It follows that $\lim _{n \rightarrow \infty} E[X(n, k)]^{2}=0$ and our first assertion is proved.

(b) If the system (30) is exponentially stable in $l_{h}$, then there exist $\beta>1$ and $\alpha \in(0,1)$ such that $\left\|y_{n}\right\|_{h} \leq \beta \alpha^{n}$ for all $n \in \mathbb{N}$. Reasoning as above we can deduce that for all $k \in \mathbb{N}$ there exists an $M_{k} \in \mathbb{R}_{+}$such that $P_{i}^{k} h^{|i|} \leq M_{k}$ for all $i \in \mathbb{Z}$. Then

$$
E[X(n, k)]^{2}=\sum_{i=-\infty}^{\infty} P_{i}^{k} h^{|i|} \frac{y_{n-k}^{i}}{h^{|i|}} \leq M_{k}\left\|y_{n-k}\right\|_{h} \leq \widetilde{M}_{k} \alpha^{n-k},
$$

where $\widetilde{M}_{k}=\beta M_{k}$. Hence the system (2) is mean square exponentially stable.

(c) If (P2) holds, then $P_{i}^{0}=\delta_{i}^{i_{0}}$ and $P_{i}^{0} h^{|i|} \leq h^{\left|i_{0}\right|}$ for $i \in \mathbb{Z}$. Defining $M_{0}=h^{\left|i_{0}\right|}$ we see that the hypotheses of statement (b) are satisfied and we obtain the conclusion. The proof is complete.

7. Concluding remarks. We have built a simple personal saving model when the bank offers interest based on fluctuation of national growth rate. The results could be easily extended to the case of stochastic equations in general separable real Hilbert spaces. We have also established connections between the stochastic stability of our model and the deterministic stability of related partial difference equations. Thus we are able to deduce asymptotic behavior of our stochastic model. Such asymptotic behavior can be compared with the real situations and provide insights for the investors to decide on their investment policies. Since we base our investigations on random walk models, there are limitations to the predictive power of our stochastic model. However, it is hoped that more realistic random models can be imposed and the corresponding saving equations studied in the near future. 


\section{References}

[1] S. S. Cheng, Partial Difference Equations, Taylor and Francis, 2003.

[2] S. S. Cheng and G. H. Lin, Green's function and stability of a linear partial difference scheme, Computers Math. Appl. 35 (1998), no. 5, 27-41.

[3] I. Gikhman and A. Skorokhod, Introduction à la théorie des processus aléatoires, Mir, Moscow, 1977.

[4] R. S. Lipster and A. N. Shiryayev, Statistics of Random Processes I. General Theory, Springer, 1977.

[5] T. Morozan, Stability and control for linear discrete-time systems with Markov perturbations, Rev. Roumaine Math. Pures Appl. 40 (1995), 471-494.

[6] V. M. Ungureanu, Uniform exponential stability for linear discrete time systems with stochastic perturbations in Hilbert spaces, Boll. Un. Mat. Ital. (8) 7 (2004), 757-772.

[7] - Uniform exponential stability and uniform observability for time-varying linear stochastic systems, in: Operator Theory: Adv. Appl. 153, Birkhäuser, Basel, 2005, 287-306.

[8] J. Zabczyk, Stochastic control of discrete-time systems, in: Control Theory and Topics in Functional Analysis (Trieste, 1974), IAEA, Vienna, 1976, 187-224.

Department of Mathematics

"Constantin Brancusi" University

Bulevardul Republicii, nr. 1, jud. Gorj

Targu Jiu, Romania

E-mail: vio@utgjiu.ro
Department of Mathematics Tsing Hua University Hsinchu, Taiwan 30043, R.O. China E-mail: sscheng@math.nthu.edu.tw

Received 5.4.2007

and in final form 19.10.2007 